\title{
The imperative of clinical and molecular research on neonatal opioid withdrawal syndrome
}

\author{
Raghu P. Metpally ${ }^{1}$ Sarath Krishnamurthy ${ }^{1} \cdot$ Karena M. Moran ${ }^{2} \cdot$ Andrew E. Weller $^{3} \cdot$ Richard C. Crist $^{3}$. \\ Benjamin C. Reiner $\mathbb{D}^{3} \cdot$ Glenn A. Doyle $\mathbb{D}^{3} \cdot$ Thomas N. Ferraro $^{4}$ - Uppala Radhakrishna ${ }^{5} \cdot$ Ray Bahado-Singh ${ }^{5}$. \\ Vanessa Troiani ${ }^{6} \cdot$ Wade H. Berrettini $\mathbb{D}^{3,7}$
}

Received: 16 July 2019 / Accepted: 2 August 2019 / Published online: 10 September 2019

(c) Springer Nature Limited 2019

During the past three decades, the opioid epidemic has developed into a major public health issue, characterized by a fivefold increase in the incidence of opioid use disorder (OUD) among adults in the United States [1]. From 1999 to 2016, a total of 231,264 men and 120,366 women died from opioid-related causes across the United States [2]. A unique characteristic of the current epidemic is its penetration into rural America [1], placing great stress on nonurban legal and medical systems, which are particularly ill-equipped to deal with increasing numbers of OUD patients. There is recent evidence that the situation is becoming increasingly dire: overdoses from opioids increased 30\% from July, 2016 to September, 2017 in 52 jurisdictions in 45 states [3]. The widespread use of prescription opioids to treat chronic, nonprogressive musculo-skeletal pain is one important factor in the increase in OUD prevalence over the past 25 years $[4,5]$. It has been estimated that $80 \%$ of new heroin users initiated illicit opioid use with a PO [6].

Wade H. Berrettini

wadeb@pennmedicine.upenn.edu

1 Department of Molecular and Functional Genomics, Geisinger, Danville, PA, USA

2 Northeastern Pennsylvania Perinatal Quality Collaborative, Geisinger, Danville, PA, USA

3 Department of Psychiatry, Perelman School of Medicine, University of Pennsylvania, Philadelphia, PA, USA

4 Cooper School of Medicine, Rowan University, Camden, PA, USA

5 William Beaumont School of Medicine, Oakland University, Oakland, USA

6 Autism and Developmental Medicine Institute, Geisinger, Lewisburg, PA, USA

7 Geisinger Clinic, Danville, PA, USA
One consequence of the fivefold increase in the incidence of OUD among adults has been a similarly large increase in the rate of infants born to mothers with active OUD [7]. Because opioid alkaloids are lipid-soluble and freely cross the placenta, these infants have neonatal opioid withdrawal syndrome (NOWS), also known as neonatal abstinence syndrome (NAS; ICD-10 code is P96.1, neonatal withdrawal symptoms from maternal use of drugs of addiction). NOWS was first identified in the 19th century as congenital morphinism [8]. Signs typically appear 18-72 h after birth, often peaking between 34 and $50 \mathrm{~h}$ after birth, and include a persistent high-pitched cry, hyper-reactivity to environmental stimuli, gastrointestinal distress, sleeplessness, increased muscle tone, exaggerated Moro response, hyperreflexia, diaphoresis, fever, ineffective sucking motions, rhinorrhea, vomiting, diarrhea, tachypnea, and seizures in the most severe cases [9]. Severity is often quantified several times daily, in a neonatal intensive care unit (NICU), using a modified Finnegan Scale [10], with scores consistently $>8$ indicating a need for pharmacotherapy with an opioid agonist. These infants often are treated in NICUs for 1-3 weeks. Treatment may include minimizing environmental stimuli (keeping the swaddled infant in a darkened quiet room with mother) and a decreasing dose schedule of an opioid, often morphine or buprenorphine [11] or methadone [12]. Polysubstance abuse often characterizes the maternal clinical history throughout pregnancy [13], bringing into question the role of these other substances in the NOWS clinical presentation.

An indication of the magnitude of the NOWS epidemic in some geographical regions of the US can be derived from electronic health record (EHR) data in northeastern and central Pennsylvania, a largely rural area characterized by few economic opportunities, poverty, and limited educational resources. Geisinger is an integrated health care system (14 hospitals and >100 primary care practice sites), with $\sim 2$ million patients. Geisinger has had an EHR since 
Table 1 ICD10 codes for mothers of NOWS babies at Geisinger (526 infants born to 473 mothers, 11/2007-4/2019)

\begin{tabular}{llll}
\hline ICD10 & ICD10 Description & Count 3 calls & \%3 calls \\
\hline O09.899 & Supervision of other high risk pregnancies, unspecified trimester & 332 & 70.19 \\
O99.320 & Drug use complicating pregnancy, unspecified trimester & 279 & 58.99 \\
F11.20 & Opioid dependence, uncomplicated & 159 & 57.72 \\
Z72.0 & Tobacco use & 152 & 33.62 \\
F32.9 & Major depressive disorder, single episode, unspecified & 151 & 32.14 \\
F19.20 & Other psychoactive substance dependence, uncomplicated & 149 \\
B18.2 & Chronic viral hepatitis C & 141 \\
Z34.80 & Encounter for supervision of other normal pregnancy, unspecified & 138 \\
Z23 & trimester & 138 \\
O09.893 & Encounter for immunization & 136 \\
F17.200 & Supervision of other high risk pregnancies, third trimester & 132 \\
O99.330 & Nicotine dependence, unspecified, uncomplicated & 126 \\
Z79.891 & Smoking (tobacco) complicating pregnancy, unspecified trimester & 29.92 \\
O09.892 & Long term (current) use of opiate analgesic & 113 \\
F19.10 & Supervision of other high risk pregnancies, second trimester & 109
\end{tabular}

\begin{tabular}{|c|c|c|c|c|c|c|c|c|c|c|c|c|c|c|}
\hline Year & 2007 & 2008 & 2009 & 2010 & 2011 & 2012 & 2013 & 2014 & 2015 & 2016 & 2017 & 2018 & 2019 & Total \\
\hline Number of Infants born with NOWS & 1 & 7 & 16 & 29 & 29 & 33 & 52 & 61 & 60 & 72 & 63 & 73 & 30 & 526 \\
\hline
\end{tabular}

The most common ICD-10 codes and description of the codes are listed for women who gave birth to infants with NOWS at Geisinger during the interval, 11/2007-4/2019. These are only those NOWS children and mothers who have been seen at Geisinger in the past 2 years, so they are active patients. The ICD-10 code count (from a total of 473 mothers) with each ICD-10 code and the percent (from a total of 473 ) is given. The term, three calls, indicates that three instances of an ICD-10 code was sufficient for that code to be included. In the bottom panel, the year of birth for the NOWS infants is listed. Note the trend for greater numbers of NOWS infants born at Geisinger in the last decade

1996. From Geisinger EHR review, there were 672 pregnant women with OUD who delivered babies at Geisinger hospitals from January 1, 2016 to August 31, 2018. This represents $5 \%$ of all live births at Geisinger for these 30 months. During this time interval, only $14 \%$ of pregnant women with OUD were in medication-assisted treatment at time of delivery [14]. Clearly, more must be done to enroll these women into medication-assisted treatment.

Little is known of the cognitive and behavioral challenges the NOWS infants face as they develop through childhood into adolescence and young adulthood, the age range (13-30 years) when most psychiatric disorders emerge. Case-control studies of early childhood development (<age $\sim 5$ ) for infants with prenatal opioid exposure have reported some cognitive, psychomotor, and behavioral deficits, when the case and control groups are carefully matched for socioeconomic status, parental educational achievement, and other relevant factors (for review see Baldacchino et al.) [15, 16]. A limitation of these data concerns the small sample sizes ( $<200$ participants) of the pooled studies. This meta-analysis showed that there were statistically significant impairments in cognitive, psychomotor, and behavioral outcomes among the opioid exposed participants $[15,16]$.
A larger study of Australian school children reported a significant and progressive cognitive deficit among NOWS children, worsening in early adolescence [17]. The authors compared NOWS children born 2000-2006 with socioeconomic and demographically matched children for national standardized test scores in grade $3(n=1661)$, grade $5(n=1104)$, or grade $7(n=499)$. In all three grades, there were highly statistically significant deficits in reading, arithmetic, grammar, spelling, and writing test scores for the NOWS children, as compared to the matched controls, with the largest deficiencies in grade 7. Among NOWS children, $29 \%$ failed to meet national minimum standards on at least one of five tests, compared with $12 \%$ of the socioeconomic and demographically matched controls. However, the control group may not have been well matched to the NOWS group for parental educational achievement, parental antenatal care, birth data (e.g., birth weight and $5 \mathrm{~min}$ APGAR score), and indigenous ancestry. These potential confounds might explain some of the group differences. Nevertheless, these data suggest that NOWS children and adolescents may need specific educational programs to help them succeed in school. Defining factors that determine susceptibility to this opioid-induced cognitive deficit is critical to identification of the vulnerable child and 
implementation of specialized education to enhance academic success.

A phenotype potentially related to the cognitive deficits among NOWS children is reduced head circumference (HC). In a recent study of 858 neonates, NOWS infants ( $n=429)$ at birth had smaller HC measurements [18]. The smaller HCs appear to persist into childhood and adolescence $[19,20]$. However, there are no longitudinal studies linking the smaller HCs at birth to cognitive deficits in childhood.

Chronic OUD is associated with decreased volumes of several brain regions, especially the frontal and temporal lobes, as assessed by structural MRI (for review see Wollman et al.) [21]. Such volume losses are present among OUD disorder patients maintained on methadone [22]. The limited data from small studies suggest regional brain volume losses (e.g., Sirnes et al.) [20] may occur in NOWS children. Whether these regional volume losses are associated with cognitive impairments is unknown.

Data are lacking on the psychiatric diagnoses that may develop among NOWS children as they progress through adolescence into young adulthood, a time when many such disorders become common. Longitudinal well-controlled studies of psychiatric diagnoses among these adolescent individuals must be conducted to guide development of prevention and early intervention strategies, leading to improved adult outcomes. This is especially critical for substance use disorders, which often begin in adolescence.

Whereas there are few data about the psychiatric diagnoses, which occur in NOWS children as they progress into young adulthood, maternal diagnoses can be made when pregnant women with OUD present to a healthcare system for childbirth medical care and for subsequent appointments. We examined ICD-10 codes for Geisinger NOWS mother-infant dyads who have been seen at least once in the past 2 years (in addition to the delivery inpatient stay) so that there are longitudinal EHR data. With this restriction, 473 pregnant women with OUD presented to Geisinger for delivery of 526 neonates between 11/2007 and 4/2019. Table 1 below shows the most common ICD-10 codes for these women. Consistent with epidemiologic data on women with OUD, there are high rates of nicotine use disorder and major depressive disorder [1]. At the bottom of Table 1, the number of NOWS infants born in that calendar year is provided. There is an ominous trend for increased numbers of NOWS infants at Geisinger over the past decade.

Pregnant women with OUD often have little or no prenatal care or OUD treatment, due to poverty, lack of access to obstetric and addiction care (especially in rural areas), mistrust of the healthcare system, concerns about legal issues and risk for incarceration. In some states, these women are committing the crime of "child endangerment" by using illicit opioids while pregnant. As a reflection of these issues, only $14 \%$ of pregnant women with OUD are on medication-assisted treatment upon presentation in labor at a Geisinger hospital. Only $45 \%$ of women with OUD attend their postpartum appointment at Geisinger, compared with $72 \%$ of women without OUD [14]. Our medical systems must do more to engage these vulnerable women in efficacious medication-assisted therapy.

Regarding genetic investigations, there have been no genome-wide association studies of NOWS. However, candidate gene papers have appeared (e.g., Wachman et al.) [23], including epigenetic studies of candidate genes [24]. Unfortunately, samples sizes of these studies are small ( $<100$ infants). There is a clear need for development of a consortium of NOWS investigators to achieve meaningful sample sizes for genome-wide approaches to NOWS genetic and epigenetic research. NIDA has begun to support the development of such consortia (https://grants.nih.gov/ grants/guide/rfa-files/RFA-DA-19-036.html).

The importance of genetic, epigenetic, and neurobiologic studies of NOWS animal models cannot be overemphasized (for review see Byrnes and Vassoler) [25]. The experimental control and accessibility of CNS tissue provides key advantages, when considering the tissue-specific epigenetic and neurobiologic effects of neonatal opioid exposure and the high probability of polysubstance abuse during all trimesters among pregnant women with OUD.

In summary, the opioid epidemic has yielded a $\sim$ fivefold increase in the incidence of NOWS infants. It is critically important that we develop a better understanding of the developmental challenges, cognitive impairments, and psychiatric syndromes, which these children will encounter, so that specialized educational modules, preventative programs, and early intervention paradigms may be developed. This will require a concerted multi-faceted research effort, including clinical studies, animal model investigations and "omics" projects. Time is short.

Acknowledgements This manuscript was produced with the support of R01 DA044015 (VT and WHB are MPIs) and a Tobacco Settlement Act grant from the Pennsylvania Department of Health (WHB is PI). RCC was supported by grant K01 DA036751. AEW was supported by T32 MH014654 (WHB is PI). BCR was supported by a 2017 NARSAD Young Investigator Award (\#26634) from the Brain and Behavior Research Foundation, as the Patrick A. Coffer Investigator, funding generously provided by Ronald and Kathy Chandonais. David Ledbetter, Ph.D., Chief Scientific Officer of Geisinger, is thanked for comments on this editorial.

\section{Compliance with ethical standards}

Conflict of interest The authors declare no conflict of interest.

Publisher's note Springer Nature remains neutral with regard to jurisdictional claims in published maps and institutional affiliations. 


\section{References}

1. Substance Absuse and Mental Health Services Administration, Office of the Surgeon General Publications and Reports of the Surgeon General. Facing addiction in America: the surgeon general's spotlight on opioids. Washington (DC): US Department of Health and Human Services; 2018.

2. Kiang MV, Basu S, Chen J, Alexander MJ. Assessment of changes in the geographical distribution of opioid-related mortality across the united states by opioid type, 1999-2016. JAMA Netw Open. 2019;2:e190040.

3. Vivolo-Kantor AM, Seth P, Gladden RM, Mattson CL, Baldwin GT, Kite-Powell A, et al. Vital signs: trends in emergency department visits for suspected opioid overdoses-United States, July 2016-September 2017. MMWR Morbidity Mortal Wkly Rep. 2018;67:279-85.

4. Brady KT, McCauley JL, Back SE. Prescription opioid misuse, abuse, and treatment in the United States: an update. Am J Psychiatry. 2016;173:18-26.

5. Cheatle MD. Facing the challenge of pain management and opioid misuse, abuse and opioid-related fatalities. Expert Rev Clin Pharm. 2016;9:751-4.

6. Compton WM, Jones CM, Baldwin GT. Relationship between nonmedical prescription-opioid use and heroin use. New Engl $\mathrm{J}$ Med. 2016;374:154-63.

7. Winkelman TNA, Villapiano N, Kozhimannil KB, Davis MM, Patrick SW. Incidence and costs of neonatal abstinence syndrome among infants with medicaid: 2004-2014. Pediatrics. 2018;141: e20173520.

8. Perlstein MA. Congenital morphinism; a rare cause of convulsions in the newborn. J Am Med Assoc. 1947;135:633.

9. Finnegan LP, Connaughton JF Jr, Kron RE, Emich JP. Neonatal abstinence syndrome: assessment and management. Addictive Dis. 1975;2:141-58.

10. Finnegan LP, Kaltenbach K. Neonatal abstinence syndrome. In: Hoekelman RA, Friedman SB, Nelson NM, editors. Primary pediatric care. Mosby: St. Louis, 1992;1367-78.

11. Kraft WK, Adeniyi-Jones SC, Chervoneva I, Greenspan JS, Abatemarco D, Kaltenbach K, et al. Buprenorphine for the treatment of the neonatal abstinence syndrome. New Engl J Med. 2017;376:2341-8.

12. Wachman EM, Minear S, Hirashima M, Hansbury A, Hutton E, Shrestha H, et al. Standard fixed-schedule methadone taper versus symptom-triggered methadone approach for treatment of neonatal opioid withdrawal syndrome. Hosp Pediatr. 2019;9:576-84.
13. Abdel-Latif ME, Oei J, Craig F, Lui K. Profile of infants born to drug-using mothers: a state-wide audit. J Paediatr Child Health. 2013;49:E80-86.

14. Moran K. Personal Communication. In: Berrettini W, editor. 2019.

15. Baldacchino A, Arbuckle K, Petrie DJ, McCowan C. Neurobehavioral consequences of chronic intrauterine opioid exposure in infants and preschool children: a systematic review and metaanalysis. BMC Psychiatry. 2014;14:104.

16. Baldacchino A, Arbuckle K, Petrie DJ, McCowan C. Erratum: neurobehavioral consequences of chronic intrauterine opioid exposure in infants and preschool children: a systematic review and meta-analysis. BMC Psychiatry. 2015;15:134.

17. Oei JL, Melhuish E, Uebel H, Azzam N, Breen C, Burns L, et al. Neonatal abstinence syndrome and high school performance. Pediatrics. 2017;139:e20162651.

18. Towers CV, Hyatt BW, Visconti KC, Chernicky L, Chattin K, Fortner KB. Neonatal head circumference in newborns with neonatal abstinence syndrome. Pediatrics. 2019;143:e20180541.

19. Walhovd KB, Westlye LT, Moe V, Slinning K, Due-Tonnessen P, Bjornerud $\mathrm{A}$, et al. White matter characteristics and cognition in prenatally opiate- and polysubstance-exposed children: a diffusion tensor imaging study. AJNR Am J Neuroradiol. 2010;31:894-900.

20. Sirnes E, Oltedal L, Bartsch H, Eide GE, Elgen IB, Aukland SM. Brain morphology in school-aged children with prenatal opioid exposure: a structural MRI study. Early Hum Dev. 2017; 106-107:33-39.

21. Wollman SC, Alhassoon OM, Hall MG, Stern MJ, Connors EJ, Kimmel CL, et al. Gray matter abnormalities in opioid-dependent patients: a neuroimaging meta-analysis. Am J Drug Alcohol Abus. 2017;43:505-17.

22. Tolomeo S, Gray S, Matthews K, Steele JD, Baldacchino A. Multifaceted impairments in impulsivity and brain structural abnormalities in opioid dependence and abstinence. Psychol Med. 2016;46:2841-53.

23. Wachman EM, Hayes MJ, Brown MS, Paul J, Harvey-Wilkes K, Terrin N, et al. Association of OPRM1 and COMT singlenucleotide polymorphisms with hospital length of stay and treatment of neonatal abstinence syndrome. Jama. 2013;309:1821-7.

24. Wachman EM, Hayes MJ, Shrestha H, Nikita FNU, Nolin A, Hoyo L, et al. Epigenetic variation in OPRM1 gene in opioidexposed mother-infant dyads. Genes, brain, Behav. 2018;17: e12476.

25. Byrnes EM, Vassoler FM. Modeling prenatal opioid exposure in animals: current findings and future directions. Front Neuroendocrinol. 2018;51:1-13. 